OPEN ACCESS

Edited by:

Shakti Singh,

University of Alberta, Canada

Reviewed by:

Veronika Lukacs-Kornek,

University of Saarland, Germany

Hiroshi Kida,

Hokkaido University, Japan

Nathan Wylie Bartlett,

University of Newcastle, Australia

*Correspondence:

David C. Jackson,

Department of Microbiology and Immunology, Peter Doherty Institute

of Infection and Immunity, The

University of Melbourne, 792 Elizabeth Street, Melbourne,

VIC 3000 , Australia

davidcj@unimelb.edu.au

Specialty section:

This article was submitted to Immunotherapies and Vaccines, a section of the journal Frontiers in

Immunology

Received: 17 April 2015 Accepted: 20 May 2015

Published: 05 June 2015

Citation:

Mifsud EJ, Tan ACL, Brown LE,

Chua BYL and Jackson DC (2015)

Generation of adaptive immune

responses following influenza virus

challenge is not compromised by pre-treatment with the TLR-2 agonist

$\mathrm{Pam}_{2}$ Cys.

Front. Immunol. 6:290.

doi: 10.3389/fimmu.2015.00290

\section{Generation of adaptive immune responses following influenza virus challenge is not compromised by pre-treatment with the TLR-2 agonist Pam $_{2}$ Cys}

\author{
Edin Jessica Mifsud, Amabel C. L. Tan, Lorena Elizabeth Brown, \\ Brendon Yew Loong Chua and David C. Jackson*
}

Department of Microbiology and Immunology, Peter Doherty Institute for Infection and Immunity, The University of Melbourne, Parkville, VIC, Australia

Immunostimulatory agents provide a new category of anti-microbial agents that activate the host's innate immune system allowing control of viral and/or bacterial infections. The TLR-2 agonist PEG-Pam 2 Cys has been shown to mediate potent anti-viral activity against influenza viruses when administered prophylactically (1). Here, we demonstrate that the treatment of mice with PEG-Pam ${ }_{2}$ Cys does not compromise their ability to generate adaptive immune responses following subsequent challenge with influenza virus. The antibody induced in mice pre-treated with $\mathrm{Pam}_{2}$ Cys possessed hemagglutinationinhibiting activities and the $\mathrm{CD}^{+}{ }^{+}$-cell responses that were elicited provided protection against heterologous viral challenge. In the absence of an effective influenza vaccine, an agent that provides immediate protection against the virus and does not compromise the induction of influenza-specific immunity on exposure to infectious virus provides an opportunity for population immunity to be achieved through natural exposure to virus.

Keywords: Pam $_{2}$ Cys, toll-like receptor-2, influenza A virus, innate immunity, adaptive immunity

\section{Introduction}

Vaccination is currently the most effective medical intervention available for prevention of influenza infection and disease. The efficacy of influenza vaccines is dependent on a match between the viral strains included in the vaccine and circulating influenza virus strains. Although anti-viral drugs can diminish symptoms and shorten the duration of illness $(2,3)$, the suggestion has been made that in the case of influenza their use can hamper the development of immunological memory (4) leaving the host susceptible to re-infection when drug treatment ceases. Although anti-influenza drugs continue to be used effectively in a clinical setting, concerns with anti-viral resistance (57) due to their widespread use have resulted in increased efforts to develop alternative prevention strategies.

Abbreviations: Ab, antibody; Influenza, influenza A virus; Pam ${ }_{2}$ Cys, S-[2,3-bis(palmitoyloxy)propyl] cysteine; TLR, toll-like receptor. 
One approach is to harness the rapid anti-microbial responses of the innate immune system, particularly for pathogens that use the respiratory portal of entry. Immunostimulatory agents provide immediate non-specific protection against virulent influenza challenge (1,8-10), and in mice, these compounds reduce the symptoms associated with influenza infection and also the viral burden reducing morbidity and promoting survival. Furthermore, by acting through the innate immune system and not directly on the pathogen, these immunostimulatory agents are unlikely to select resistant virus variants.

Many immunostimulatory agents are agonists of toll-like receptors (TLR) and mediate anti-viral and anti-bacterial activity by activating inflammatory pathways $(1,8-10)$. An understanding of the way in which these compounds affect acquisition of adaptive immunity and immunological memory is important because they could be employed for use during influenza pandemics where there is a high risk of re-exposure to pathogen. In the case of respiratory diseases, delivery of TLR agonists to the pulmonary tract can alter the immune environment, which could influence subsequent induction of adaptive immunity. An understanding of the way in which immunostimulatory agents, including TLR agonists, affect development of pathogen-specific immunity is therefore important.

We have developed a soluble form of the synthetic analog, $S$ [2,3-bis(palmitoyloxy)propyl] cysteine ( $\left.\mathrm{Pam}_{2} \mathrm{Cys}\right)$, which acts as an agonist of toll-like receptor-2 (TLR-2) and provides immediate protection against challenge with a lethal dose of influenza virus A/PR/8/34 (PR8, H1N1) (1). We have also shown that although $\mathrm{Pam}_{2}$ Cys treatment significantly reduces viral burden, reduces disease symptoms, and prevents death, it does not totally abrogate infection. This property provides the potential to develop immunity to influenza virus through asymptomatic natural infection.

In this study, we show that $\mathrm{Pam}_{2}$ Cys prophylaxis permits development of a robust influenza virus-specific adaptive immune responses comprised $\mathrm{CD} 8^{+}$T-cells, $\mathrm{CD} 4^{+}{ }^{+}$-cells, and antibodies. We also show that this virus-independent stimulation of the innate immune system does not compromise the development of heterologous immunity. The development of an agent that can provide the host with immediate protection and does not hinder the development of pathogen-specific immunity following exposure to infectious virus provides an opportunity for population immunity to be achieved through natural exposure to the virus.

\section{Materials and Methods}

\section{Synthesis of PEG-Pam ${ }_{2}$ Cys}

$\mathrm{Pam}_{2} \mathrm{Cys}$ is hydrophobic and insoluble in physiological media; therefore, we synthesized the agonist with a polyethylene glycol molecule attached to confer solubility and allow administration by the intranasal (i.n.) route. PEG-Pam ${ }_{2}$ Cys was synthesized in house using Fmoc-based chemistry as described previously (1). The product was purified by reversed-phase high-performance liquid chromatography (HPLC) using a C4 VYDAC column $(10 \mathrm{~mm} \times 250 \mathrm{~mm}$; Alltech, NSW, Australia) installed in a Waters HPLC system (Waters Millipore, Milford, MA, USA). The purity of PEG-Pam ${ }_{2}$ Cys was determined by HPLC using a VYDAC C8 column $(4.6 \mathrm{~mm} \times 250 \mathrm{~mm})$ installed in a Waters system and was found to be $>95 \%$. The authenticity of the product was determined by mass analysis (mass value found 1,502.2 Da; expected mass 1,502.1 Da) using an Agilent 1100 Series LC/MSD ion-trap mass spectrometer (Agilent, Palo Alto, CA, USA).

\section{Animals}

Female C57BL/6 mice (6-12 weeks old) were bred and housed in the Animal house facility at the Department of Microbiology and Immunology, The University of Melbourne. All animal experiments were conducted with approval from the University of Melbourne Animal Ethics Committee.

\section{Inoculations and Viral Challenge}

Mice were anesthetized using isoflurane inhalation and received either $20 \mathrm{nmol}$ of PEG-Pam 2 Cys dissolved in saline or saline alone by the i.n. route.

Mice were challenged with a virulent strain of influenza virus 3 days after treatment with PEG-Pam ${ }_{2}$ Cys. 200 plaque forming units (PFU) of the H1N1 influenza virus A/Puerto Rico/8/34 (PR8, Mount Sinai) were administered by the i.n. route during isoflurane anesthesia. Infection following challenge with PR8 in this way results in weight loss, labored breathing, and a hunched posture; animals were monitored daily for any signs of illness and euthanized at a pre-determined humane endpoint.

Challenge with less virulent strains of influenza virus were also carried out during isoflurane anesthesia with either (i) $10^{4.5} \mathrm{PFU}$ of the H3N1 virus Mem/Bel (a genetic reassortant of A/Memphis/1/71 [H3N2] × A/Bellamy/42 [H1N1]) (ii) $10^{4.5} \mathrm{PFU}$ of Mem71 A/Memphis/1/71, an H3N2 strain or (iii) $10^{4.5} \mathrm{PFU}$ of the $\mathrm{H} 3 \mathrm{~N} 2$ virus, $\times 31$. Each virus preparation was diluted in PBS and administered i.n. 3 days after receiving PEG-Pam ${ }_{2}$ Cys.

\section{Preparation of Lung Cells}

Following $\mathrm{CO}_{2}$ asphyxiation, lungs were removed and subjected to enzymatic digestion with collagenase A $(2 \mathrm{mg} / \mathrm{ml}$; Roche, Mannheim, Germany) in RPMI 1640 medium for 30 minutes $37^{\circ} \mathrm{C}$. Cells were strained through a metal sieve and treated with ammonium-Tris hydrochloride $(7.4 \% \mathrm{w} / \mathrm{v}$ ammonium chloride, $2.06 \% \mathrm{w} / \mathrm{v}$ Tris hydrochloride [ATC]) for $5 \mathrm{~min}$ at $37^{\circ} \mathrm{C}$ and were then washed twice with RP-10 (RPMI 1640 containing $10 \%$ fetal calf serum, $2 \mathrm{mM}$ L-glutamine, $76 \mathrm{mM}$ 2-meceptoethanol, $150 \mathrm{U} / \mathrm{ml}$ penicillin, $150 \mathrm{mg} / \mathrm{ml}$ streptomycin, $150 \mathrm{mM}$ nonessential amino acids [all supplements were obtained from Life Technologies]) in $7.5 \mathrm{mM}$ HEPES. The number of viable cells was determined using trypan blue exclusion.

\section{Intracellular Cytokine Staining}

Lung and spleen cells $\left(2-3 \times 10^{6} / 200 \mu \mathrm{l}\right)$ were stimulated in the presence or absence of peptide $\mathrm{PA}_{224-236}$ or $\mathrm{NP}_{366-374}(1 \mu \mathrm{g} / \mathrm{ml})$ with $5 \mu \mathrm{g} / \mathrm{ml}$ of GolgiPlug (BD Biosciences Pharmingen) $25 \mathrm{U} / \mathrm{ml}$ recombinant human IL-2 (Roche, Indianapolis, IN, USA) for $6 \mathrm{~h}$ at $37^{\circ} \mathrm{C}$. Following stimulation, cells were stained with PercP5.5 antimouse $\mathrm{CD} 8 \alpha$ (BD Biosciences Pharmingen) then fixed and permeabilized using the BD Cytofix/Cytoperm kit (BD Biosciences Pharmingen) according to the manufacturer's instructions. Cells were finally stained with FITC-labeled antibody directed against interferon- $\gamma($ IFN- $\gamma)$, Pe-Cy7-labeled antibodies directed against 
tumor necrosis factor- $\alpha$ (TNF- $\alpha$ ) and APC-labeled antibodies directed against IL-2. Samples were analyzed using a FACSCanto II and analyzed using FlowJo Software.

\section{Hemagglutination Inhibition Assay}

Sera were prepared from blood and stored at $-20^{\circ} \mathrm{C}$ until use. To remove any non-specific inhibitors of hemagglutination, sera were diluted $1 / 5$ with receptor destroying enzyme (RDE II, Denka Seiken Co., Ltd.) and incubated at $37^{\circ} \mathrm{C}$ overnight. Sodium citrate $(1.6 \% \mathrm{w} / \mathrm{v}$ from Merck; Kilsyth, Victoria) diluted in PBS (Media Preparation Facility, Department of Microbiology and Immunology). $0.1 \%$ sodium citrate (Chem Supply) was added and samples were incubated for a further $2 \mathrm{~h}$ at $56^{\circ} \mathrm{C}$ prior to use. The hemagglutination inhibition (HI) assay was performed using either Mem/Bel or PR8 according to the method described in Ref. (11) and modified to a micro-titer format.

\section{In vivo Cytotoxic T-Cell Assay}

An in vivo cytotoxic T-cell (CTL) assay was performed in mice that had been primed with Mem71 virus and challenged 1 month later with PR8 virus using a previously described method (12). The data generated were analyzed using FlowJo software and the percentage specific lysis of CFSE-labeled target cells in each mouse calculated using the following equation:

$$
\begin{gathered}
\% \text { specific lysis }=1-\frac{r(\text { non-infected })}{r(\text { infected })} * 100 \\
\text { where } \mathrm{r}=\% \frac{\text { CFSE low }}{\text { CFSE high }}
\end{gathered}
$$

\section{CD4 $^{+}$T-Cell IFN- $\gamma$ Detecting ELISPOT}

Membrane-based 96-well plates (MAIPS4510; Millipore, North Ryde, NSW, Australia) were coated with anti-mouse IFN- $\gamma$ capture antibody (clone R4-6A2; Pharmingen) prior to addition of $5 \times 10^{5}$ cells to each well followed by $50 \mu \mathrm{l} \mathrm{NP} 311-325$ peptide $(5 \mu \mathrm{g} /$ well). Four wells lacking peptide were included as negative controls. Cells were cultured for $18 \mathrm{~h}$ at $37^{\circ} \mathrm{C} 5 \% \mathrm{CO}_{2}$ and IFN- $\gamma$ detected using biotinylated mouse anti-IFN- $\gamma$ detection $\mathrm{Ab}$ (clone XMG1.2; Pharmingen) and streptavidin-alkaline phosphatase (Pharmingen) as described elsewhere (13). Spots formed by the deposition of enzyme substrate were counted using an ELISPOT plate reader (AID Autoimmun Diagnotika, Strassberg, Germany) and analyzed using AID software. The number of spotforming units (SFU) was calculated by subtracting the sum of the background value plus two SD and responses considered positive when the net SFU value was $>20 \mathrm{SFU} / 10^{6}$ cells.

\section{Determination of Influenza Virus Titers}

Lung viral titers were determined using an MDCK cell-based plaque assay as previously described (14).

\section{Characterization of the Pulmonary Cytokine Environment}

The levels of IFN- $\gamma$, TNF- $\alpha$, interlukin-6 (IL-6), IL-10, IL12 p70, and monocyte chemoattractant protein-1 (MCP-1) in bronchoalveolar lavage (BAL) fluid or lung tissue were analyzed using a BD Cytometric Bead Array (CBA) Mouse Inflammation Kit (BD Biosciences, San Diego, CA, USA) according to the manufacturer's instructions with the exception that a total of $2 \mu \mathrm{l}$ of each capture bead was used in $50 \mu \mathrm{l}$ of BAL sample and the PEdetection reagent was diluted 1 in 5 . Samples were analyzed using a Becton Dickinson FACSCalibur flow cytometer and data analyzed using the FlowJo software package (Tree Star, Inc., Ashland, OR, USA).

\section{Statistical Analyses}

For comparison of two data sets, a two-tailed Student's $t$-test was used. For comparison of data sets with a non-Gaussian distribution, a Mann-Whitney $t$-test was used. A $P$-value $\leq 0.05$ was considered statistically significant. Statistical analyses were carried out using the GraphPad Prism 6 software package.

\section{Results}

\section{Adaptive Immune Responses Generated Following Challenge with Virulent and Non-Virulent Influenza Virus Strains}

In the C57BL/6 mouse model of influenza infection, the neutralizing $\mathrm{Ab}$ response is directed predominantly to the viral hemagglutinin and the $\mathrm{CD} 8^{+}$T-cell response is directed to the two immunodominant epitopes $\mathrm{PA}_{224-236}$ and $\mathrm{NP}_{366-374}$, present on the internal proteins, acid polymerase and nucleoprotein, respectively (15).

Use of non-virulent Mem-Bel (H3N1) influenza virus allowed us to follow the adaptive immune response beyond 7 days, the time point at which mice infected with virulent PR8 (H1N1) influenza virus succumb to infection. Saline or PEG-Pam ${ }_{2}$ Cys was administered to mice 3 days prior to challenge with $10^{4.5} \mathrm{PFU}$ of Mem-Bel virus (Figure 1A), mice were monitored daily following influenza challenge and weight loss is shown in Figure 1B. Animals that received PEG-Pam 2 Cys maintained their overall bodyweight throughout the duration of infection, whereas animals treated with saline lost a small amount of weight during influenza challenge but regained weight 10 days after influenza challenge. The viral load in mice treated with PEG-Pam ${ }_{2}$ Cys was significantly reduced $(>1 \mathrm{log}$ ) during the early stages of infection and by day 7 , virus was cleared from the lungs of both treatment groups (Figure 1C).

No significant differences in titers of $\mathrm{HI}$ antibodies $(P=0.4977)$ were detected in the sera of saline and PEG-Pam ${ }_{2}$ Cys-treated animals 10 days after challenge (Figure 1D). Epitope-specific CD8 ${ }^{+}$ T-cell responses were examined 10 days postinfection, and no significant differences were detected in cytokine secreting $\mathrm{CD} 8^{+}$ $\mathrm{T}$-cells present in the lungs or spleens regardless of the treatment received (Figures 1E-G). Gating strategy used to identify cytokine secreting cells is shown in Figure S1 in Supplementary Material. We also detected no significant $(P=0.7381)$ numbers of $\mathrm{CD} 4{ }^{+}$T-cells in the spleens of each treatment group (Figure $\mathbf{1 H}$ ). Taken together, the results suggest that neither cell-mediated nor humoral immune responses were compromised by pre-treatment with PEG-Pam 2 Cys.

To determine the effects of PEG-Pam ${ }_{2}$ Cys on infection with a more virulent influenza virus, mice were treated with saline or the 


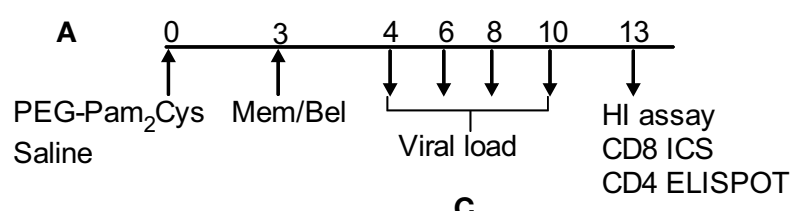

B

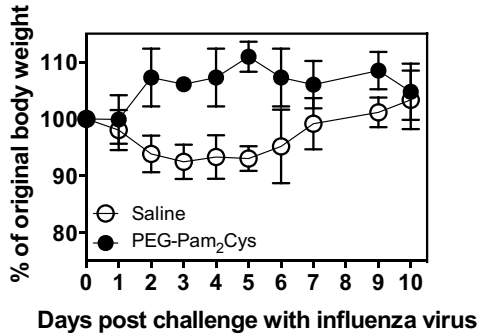

D
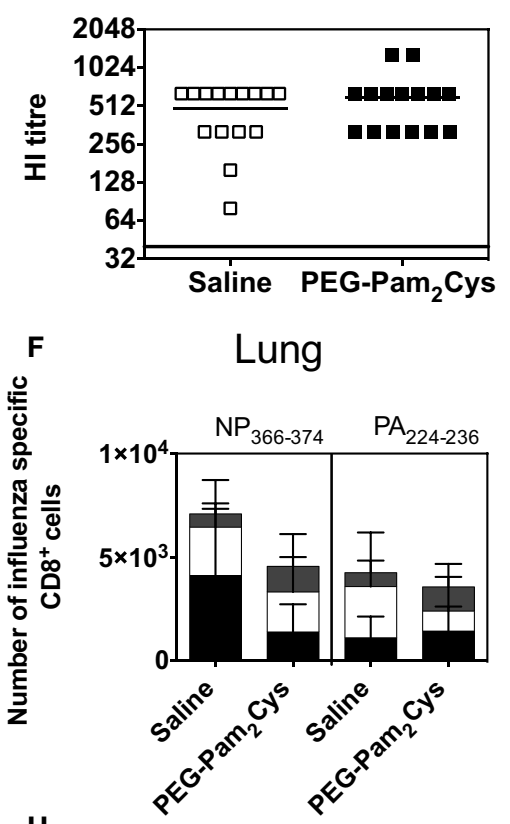

H

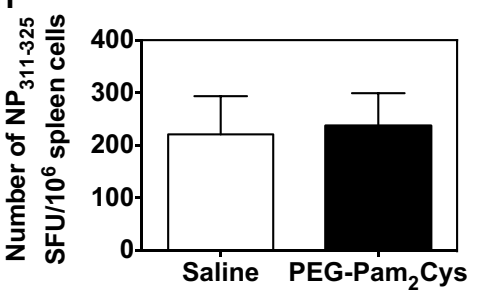

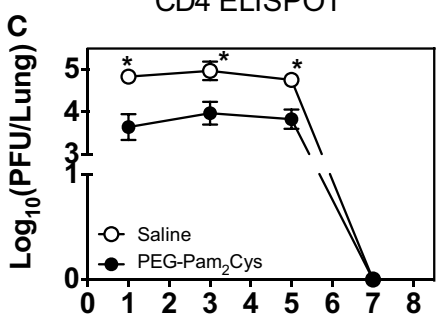

Days post challenge with influenza virus

\section{E}
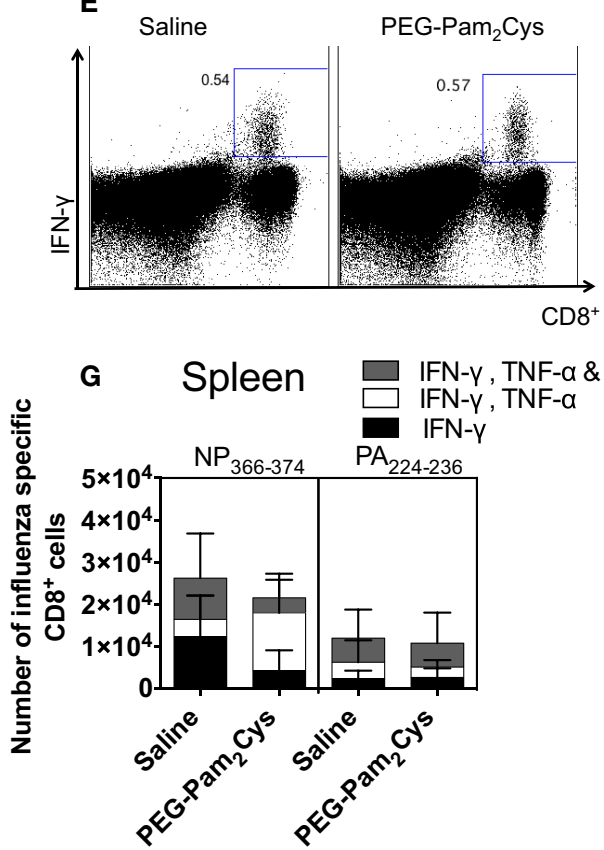

FIGURE 1 | Antibody and cell mediated immune responses generated in PEG-Pam ${ }_{2}$ Cys-treated mice following infection with Mem/Bel.

(A) Timeline of protocols used; C57BL/6 $(n=5-15)$ mice were inoculated via the i.n. route with saline or $20 \mathrm{nmol}$ of PEG-Pam ${ }_{2}$ Cys 3 days prior to challenge with $10^{4.5} \mathrm{PFU}$ of Mem/Bel. At various times following challenge, mice were culled and various virological and immunological parameters measured. (B) Percentage weight change following influenza challenge. (C) Mean viral loads present in the lung throughout the course of infection.
(D) Influenza-specific Ab levels in sera were determined using $\mathrm{HI}$ assays. (E) Representative FACS plots of the $\mathrm{NP}_{366-374}$-specific response in the lungs of PEG-Pam ${ }_{2}$ Cys and saline-treated mice. Numbers of $\mathrm{NP}_{366-374}$ and $\mathrm{PA}_{224-236}$ specific cytokine secreting $\mathrm{CD}^{+}{ }^{+}$T-cells in (F) lungs and (G) spleen. (H) Number of IFN- $\gamma$ secreting CD4 ${ }^{+}$T-cells [spot-forming units (SFU) per $10^{6}$ cells] in spleen cells following stimulation with peptide epitope $\mathrm{NP}_{311-325}$. Statistical significance for $(\mathbf{B})$ was determined using an unpaired Mann-Whitney $t$-test $\left({ }^{\star} P<0.05\right)$. immunostimulant and subsequently challenged with a lethal dose of PR8 virus (Figure 2A). Saline control mice suffered substantial weight loss and reached the previously determined humane end point 8 days after challenge (Figure $2 \mathbf{B}$ ). In contrast, mice pretreated with PEG-Pam ${ }_{2}$ Cys all survived viral challenge, a result which is consistent with our previous findings (1). 


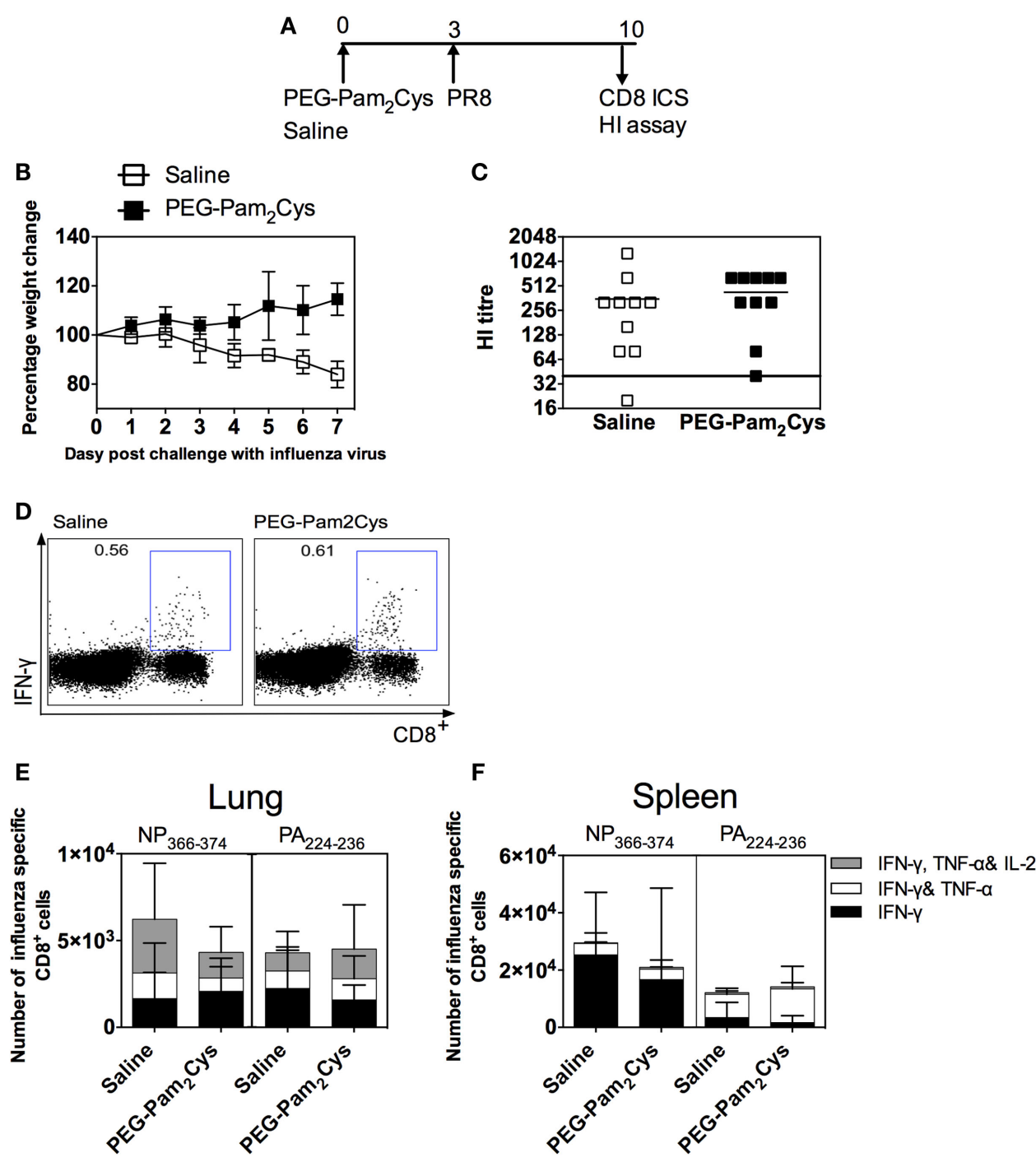

FIGURE 2 | Antibody and cell mediated immune responses generated in PEG-Pam ${ }_{2}$ Cys-treated mice following challenge with 200 PFU of PR8 influenza virus. (A) Timeline of protocols used;

C57BL/6 $(n=5-10)$ mice were inoculated via the i.n. route with saline or $20 \mathrm{nmol}$ of PEG-Pam ${ }_{2}$ Cys 3 days prior to challenge with 200PFU of PR8.

(B) Mice were monitored daily following viral challenge and the percentage change in body weight recorded. (C) Hemagglutination inhibiting Ab levels in sera. (D) Representative FACS plots of the $\mathrm{NP}_{366-374}$-specific CD8 ${ }^{+}$T-cell response in lungs. Numbers of $\mathrm{NP}_{366-374}$ or $\mathrm{PA}_{224-236}$ specific cytokine secreting $\mathrm{CD}^{+}{ }^{+}$T-cells in $(\mathbf{E})$ lungs and $(\mathbf{F})$ spleen. Statistical significance was determined using an unpaired Student's $t$-test $\left({ }^{\star} P<0.05\right)$.
Another group of C57BL/6 mice treated with either saline or PEG-Pam 2 Cys and subsequently challenged with PR8 were euthanized 7 days after viral challenge to asses the adaptive immune responses. Non-significant titers of $\mathrm{HI}$ antibodies $(P=0.2607)$ were detected in sera of animals 7 days after challenge with PR8 virus (Figure 2C) whether they had been pre-treated with saline. When the fine specificity of the $\mathrm{CD}^{+} \mathrm{T}$-cell response was examined, very few differences were detected in the cytokine profiles of $\mathrm{PA}_{224-236}$ and $\mathrm{NP}_{366-374}$-specific CD8 ${ }^{+}$T-cells obtained from lungs and spleen of mice whether treated with saline or PEG$\mathrm{Pam}_{2} \mathrm{Cys}$ (Figures 2D-F). The results again indicate that treatment with PEG-Pam ${ }_{2}$ Cys has little or no deleterious effect on the development of adaptive immune responses.

\section{PEG-Pam ${ }_{2}$ Cys Treatment does not Affect the Development of Recall CD8 T-Cell Responses or the Development of Heterologous Immunity}

Because $\mathrm{CD}^{+}$T-cells target the internal, conserved epitopes of the influenza virus proteins, they are able to recognize a broad range of influenza strains (16). These cells are, however, short lived and require constant stimulation for persistence. The use of Mem'71 (H3N2) results in a resolving infection, which allowed us to track the maintenance of influenza-specific immune responses and also to determine the ability to provide protection against a second challenge with a heterologous strain of virus.

To examine the long-term functional and cross-protective capabilities of the $\mathrm{CD} 8^{+}$T-cell response generated, we assessed $\mathrm{CD} 8^{+}$ 


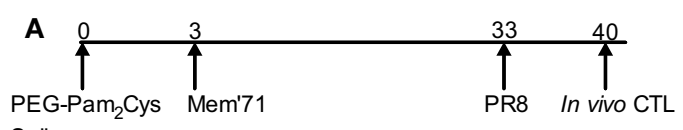

Saline

B

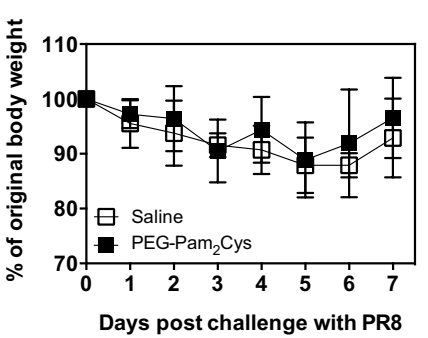

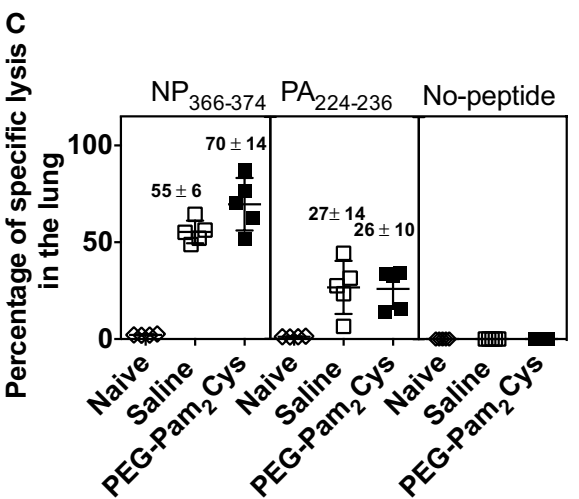

FIGURE 3 | Influenza-specific cytotoxic CD8 ${ }^{+}$T-cells persist in the lung and the spleen of PEG-Pam $\mathbf{2}_{\mathbf{2}}$ Cys-treated mice. (A) Time line of protocol used; C57BL/6 mice $(n=5)$ received saline or PEG-Pam ${ }_{2}$ Cys 3 days prior to challenge with $10^{4.5} \mathrm{PFU}$ of Mem71 influenza virus. One month later, mice were challenged with a lethal dose of PR8. (B) Percentage weight change after secondary influenza challenge. Seven days after challenge with PR8 naïve "donor" splenic cells were differentially labeled with CFSE and pulsed with no peptide, peptide $\mathrm{NP}_{366-374}$, or peptide $\mathrm{PA}_{224-236}$ before intravenous transfer via

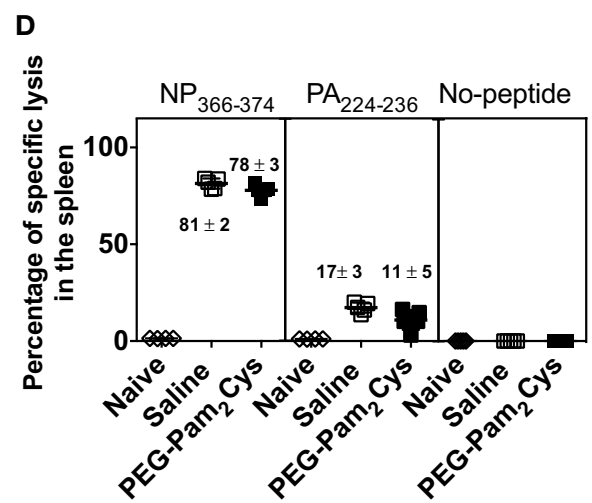

the base of tail into recipient mice. Recipient mice were killed and remaining labeled donor cells in the lungs and spleens enumerated using flow cytometry. The percentage of specific lysis observed in the lung (C) and spleen (D) are shown. Each symbol in (C,D) represents the percentage of specific lysis obtained by individuals and the vertical line indicates the mean of each group. Numbers above each group indicate the mean amount of specific lysis of each groups with the SD. Data are from one of the two independent experiments, which yielded similar results.
T-cell responses using an in vivo CTL assay. Following treatment with PEG-Pam ${ }_{2}$ Cys or saline and subsequent challenge with Mem'71 (H3N2) virus, mice were then challenged 4 weeks later with a lethal dose of the heterologous PR8 (H1N1) virus (Figure 3A). The results (Figure 3B) demonstrate that both groups were protected from lethal PR8 challenge, which typically causes $20 \%$ weight loss by day 7 (Figure 2B), indicating that treatment with Pam2Cys does not compromise the ability to elicit and maintain immunity against heterologous virus challenge.

Seven days after secondary infection splenocytes from naïve, "donor" mice were pulsed with either $\mathrm{PA}_{224-236}$ peptide, $\mathrm{NP}_{366-374}$ peptide or received no treatment. The cells were then differentially labeled with different concentrations of CFSE and injected intravenously via the base of tail into recipient mice. After $14 \mathrm{~h}$, labeled cells present in lungs and spleen were enumerated by flow cytometry and the gating strategy is shown in Figure S2 in Supplementary Material. The difference in the number of CFSE-labeled cells in infected mice compared to uninfected mice revealed that the $\mathrm{CD}^{+}$T-cell response generated in mice pretreated with PEG-Pam ${ }_{2}$ Cys or saline were equally effective at killing donor cells (Figures 3C,D). The results clearly demonstrate that prophylaxis with PEG-Pam ${ }_{2}$ Cys did not compromise the function or quality of the $\mathrm{CD} 8^{+} \mathrm{T}$-cell response generated. The results of the experiments further demonstrate that the immunostimulatory effects of PEG-Pam ${ }_{2}$ Cys do not affect the cytotoxic capabilities of T-cells responsible for influenza-specific immunity.

To further characterize the $\mathrm{CD}^{+}$T-cell response, the cellular cytokine profiles were examined by ICS (Figure 4A) and the gating strategy is shown in Figure S3 in Supplementary Material. There were no significant differences in the numbers of $\mathrm{PA}_{224-236}$ or $\mathrm{NP}_{366-374}$-specific T-cells capable of secreting a combination of cytokines in the lungs and spleens of saline and PEG-Pam 2 Cys treatment groups (Figures 4B-D). These results confirm our earlier findings (1) that Pam2Cys does not hinder development of influenza-specific immune responses. We now show that the influenza-specific immune response can be recalled by secondary infection with a different influenza virus and that these cells possess cytolytic function and secrete a combination of cytokines associated with protection. 


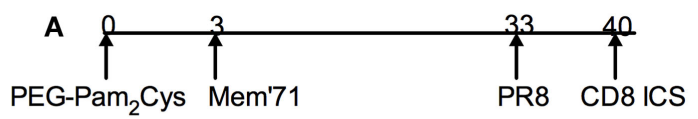

Saline
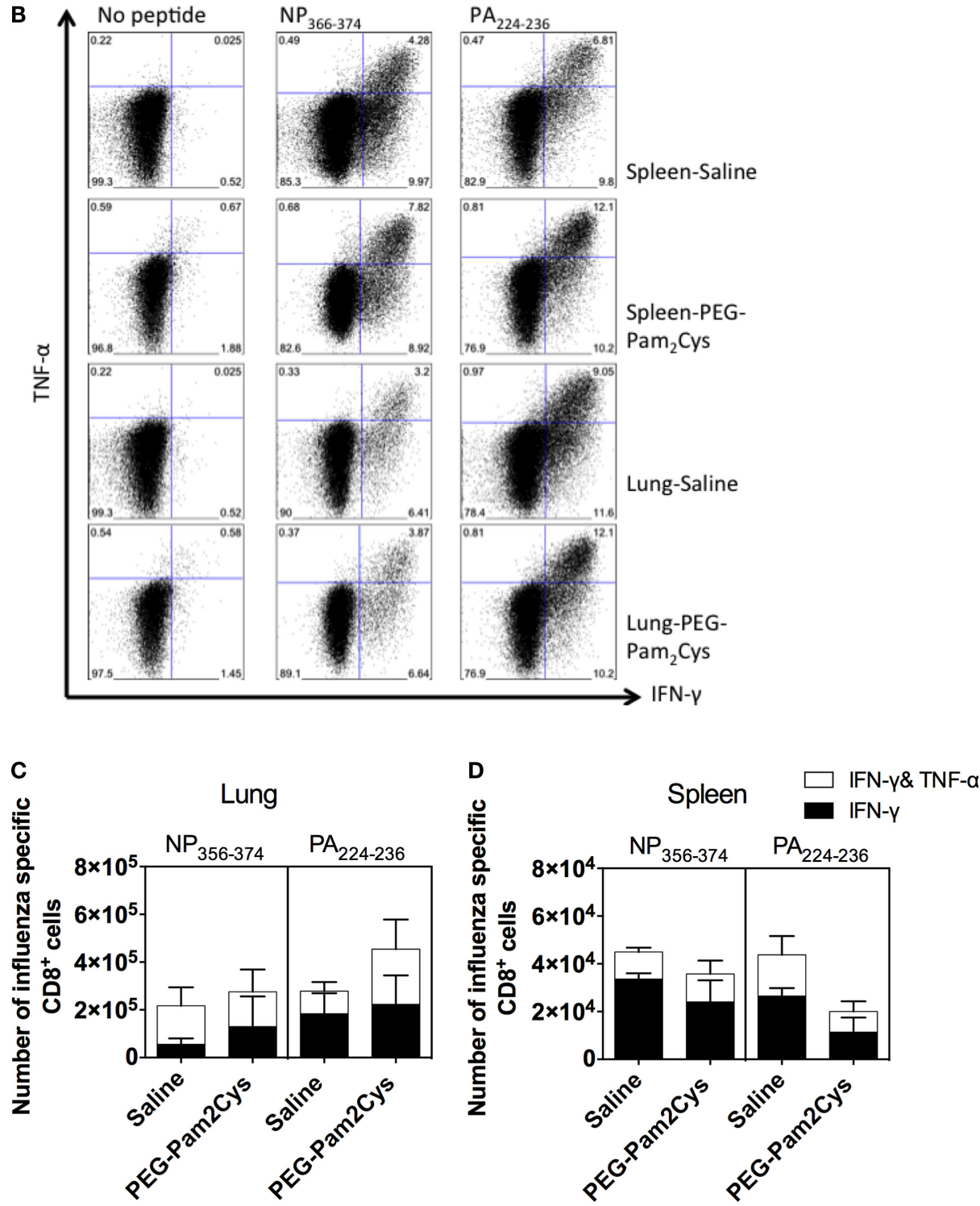

FIGURE 4 | Influenza-specific CD8 ${ }^{+} \mathrm{T}^{-}$-cell responses persist in the spleen and lung following stimulation with PEG-Pam ${ }_{2}$ Cys. (A) Timeline of protocol used; C57BL/6 mice $(n=5)$ received 20 nmol of PEG-Pam 2 Cys or saline 3 days prior to challenge with $10^{4.5} \mathrm{PFU}$ of Mem71. One month after primary challenge, mice were challenged with PR8 influenza virus, and 7 days later, an ICS assay was performed to examine the cytokine profile of influenza-specific CD8 ${ }^{+}$T-cells that were generated. (B) Representative FACS plots show the percentage of $\mathrm{CD}^{+}{ }^{+}$T-cells from the spleen secreting either IFN- $\gamma$ and/or TNF- $\alpha$. Numbers of $\mathrm{NP}_{366-374}$ or $\mathrm{PA}_{224-236}$ specific CD8 ${ }^{+}$T-cells secreting cytokines in lung (C) and spleen (D). Results are expressed as the mean ( $\pm 1 \mathrm{SD}$ ). Data are from one of the two independent experiments that yielded similar results.

\section{Pam $_{2}$ Cys Treatment does not Alter Adaptive Immune Responses Generated in Immunologically Experienced Mice}

Following vaccination or natural infection, human beings are no longer immunologically naïve. If immunostimulatory agents are to be used in human beings, we need to determine whether or not they affect existing antigen specific T-cells. Others $(17,18)$ have shown that subsequent and heterologous influenza virus infections cause an influx of $\mathrm{CD}^{+}$T-cells into lungs. These infections, or more specifically the inflammation that they induce, 

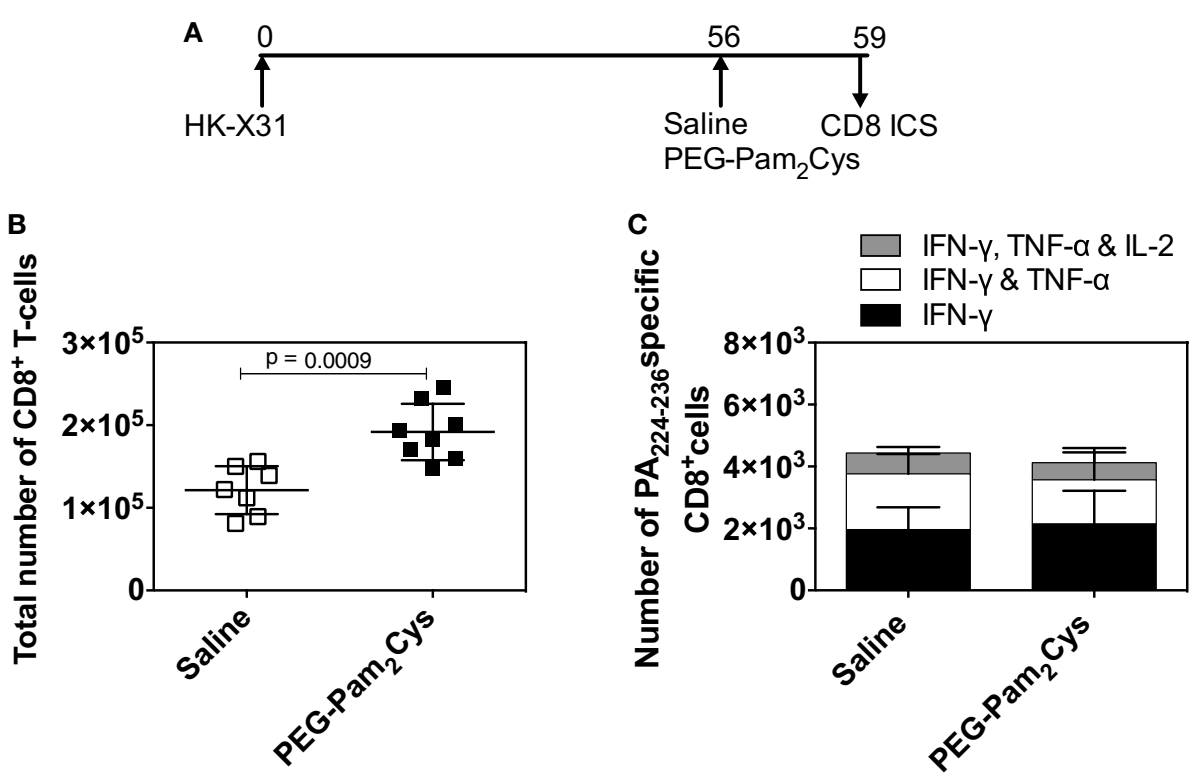

FIGURE 5 | PEG-Pam ${ }_{2}$ Cys treatment alone does not activate memory CD8 $^{+}$T-cells. (A) Timeline of protocol used; C57BL/6 mice were challenged with $10^{4.5} \mathrm{PFU}$ of $\mathrm{X} 31$ virus, and 2 months later, mice received either saline or $20 \mathrm{nmol}$ of PEG-Pam ${ }_{2}$ Cys. After 3 days, mice were euthanized and the $\mathrm{CD}^{+}{ }^{+} \mathrm{T}$-cell responses assessed. (B) Total number of $\mathrm{CD}^{+}{ }^{+}$T-cells in the lung. (C) Numbers of $\mathrm{PA}_{224-236}$ specific CD8 ${ }^{+}$T-cells secreting IFN- $\gamma$, IFN- $\gamma$, and TNF- $\alpha$ or IFN- $\gamma$, TNF- $\alpha$, and $\mathrm{IL}-2$ in the lung. Results are expressed as the mean ( $\pm 1 \mathrm{SD})$. Statistical significance is denoted * on the graph and was determined using an unpaired Student's $t$-test $(P<0.05)$. can lead to the recruitment of cells into the lung (19). What we have previously observed following treatment of immunologically naive animals with PEG-Pam ${ }_{2}$ Cys (1) is an increase in the numbers of $\mathrm{CD}^{+}{ }^{+}$-cells. We therefore determined whether or not PEG-Pam 2 Cys delivered intanasally affected resident $\mathrm{CD}^{+}{ }^{+} \mathrm{T}-$ cells elicited by previous infection. Immunologically experienced mice were generated by challenge with a non-lethal dose of X31 influenza virus and 2 months later, mice were treated with saline or PEG-Pam ${ }_{2}$ Cys (Figure 5A). We observed an increase in the number of $\mathrm{CD}^{+}$T-cells in lungs of mice treated with PEG$\mathrm{Pam}_{2}$ Cys (Figure 5B) supporting our earlier observations (1) but did not observe significant differences in the number and cytokine profiles of $\mathrm{PA}_{224-236}$ specific $\mathrm{CD} 8^{+}$T-cells (Figure 5C) suggesting that $\mathrm{Pam}_{2}$ Cys treatment does not activate memory CD8 ${ }^{+}$ T-cells.

We next determined whether PEG-Pam ${ }_{2}$ Cys altered the ability of mice to recall a previous immune state. Using the treatment regime shown in Figure 6A, the $\mathrm{PA}_{224-236}$ specific $\mathrm{CD} 8^{+} \mathrm{T}$-cell response was examined 7 days after challenge with PR8 virus. Comparable numbers of $\mathrm{CD}^{+}$T-cells secreting IFN- $\gamma$ alone, IFN- $\gamma$ plus TNF- $\alpha$, or IFN- $\gamma$ plus TNF- $\alpha$ plus IL- 2 were detected in the lungs of mice (Figure 6B). Taken together, the data suggest that stimulation of the innate immune system with PEG-Pam 2 Cys does not impact secondary recall responses.

\section{Discussion}

In this study, we have shown that PEG-Pam 2 Cys treatment provides the host with immediate defense against influenza by reducing viral burden, eliminating disease symptoms, and also allows the generation of adaptive immune responses that are quantitatively and qualitatively similar to those generated during natural infection.

The cellular and humoral arms of the adaptive immune system each combat influenza virus infection and both mitigate disease severity. It is therefore necessary that immunomodulatory agents developed to target influenza should not hinder the development of either arm of the adaptive immune response. Even though PEG$\mathrm{Pam}_{2}$ Cys treatment causes a dramatic reduction ( $\left.90 \%\right)$ in antigen load compared with treatment with saline, similar numbers of $\mathrm{CD}^{+}$T-cells were elicited. This is unexpected given previous findings (20-22) that decreased antigen loads have profound effects on resulting T-cell responses. Possible explanations for this are the enhanced proliferation of $\mathrm{CD}^{+} \mathrm{T}$-cells, due to their expression of TLR-2, following stimulation with TLR-2 ligands even in the absence of co-stimulation by APCs (23). Direct activation of TLR-2 has also been shown to reduce the amount of antigen required for $\mathrm{CD} 8{ }^{+} \mathrm{T}$-cell activation even promoting proliferation of $\mathrm{CD}^{+}$T-cells with low TCR and MHC affinity (24). It seems then that TLR-2-mediated stimulation of $\mathrm{CD}^{+}{ }^{+} \mathrm{T}$-cells decreases or even obviates the need for co-stimulation by APC improving the chances for successful $\mathrm{CD}^{+} \mathrm{T}$-cell responses even in the presence of reduced antigen and low affinity TCR. Secondly, inflammation has been shown by many groups to play a crucial role in the contraction phase and development of memory $\mathrm{CD}^{+}$ T-cell responses (25-27). The inflammatory milieu induced by the pathogen has been found to be essential for maximal $\mathrm{CD}^{+}$ T-cell expansion and is also crucial for the development of effector functions such as cytolysis (28). Furthermore, Richer and colleagues (29) have shown that inflammatory cytokines reduce 


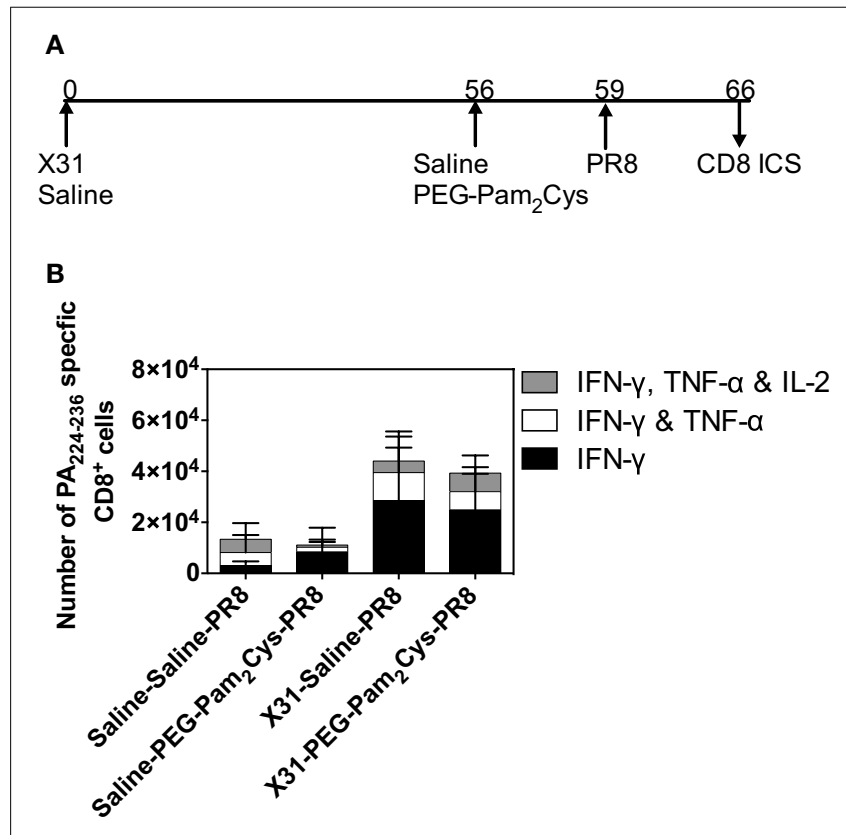

FIGURE 6 | Pre-existing adaptive immune responses are not compromised by stimulation with Pam $\mathbf{P}_{\mathbf{2}}$ Cys. (A) Timeline of infection protocol used; C57BL/6 mice $(n=5)$ were challenged with $10^{4.5}$ PFU of $\times 31$ virus, and 2 months later, mice received either saline or $20 \mathrm{nmol}$ of PEG-Pam 2 Cys 3 days prior to challenge with a lethal dose of PR8 virus. Mice were monitored daily following challenge and the $\mathrm{PA}_{224-236}$ specific $\mathrm{CD}^{+}{ }^{+}$T-cells secreting cytokine in the lung are shown in (B). Results are expressed as the mean $( \pm 1 \mathrm{SD})$

antigen sensitivity in both effector and memory $\mathrm{CD}^{+}{ }^{+} \mathrm{T}$-cell responses.

The development of prophylactic agents that augment the host's innate immune system could considerably decrease the morbidity and mortality rates that are associated with influenza pandemics for which no vaccines are available or in those cases where available vaccines are ineffective, e.g., during the $2009 \mathrm{H} 1 \mathrm{~N} 1$

\section{References}

1. Tan ACL, Mifsud EJ, Zeng W, Edenborough K, McVernon J, Brown LE, et al. Intranasal administration of the TLR2 agonist Pam2Cys provides rapid protection against influenza in mice. Mol Pharm (2012) 9:2710-8. doi:10.1021/ mp300257x

2. Aoki FY, Macleod MD, Paggiaro P, Carewicz O, El Sawy A, Wat C, et al. Early administration of oral oseltamivir increases the benefits of influenza treatment. J Antimicrob Chemother (2003) 51:123-9. doi:10.1093/jac/dkg007

3. Schirmer P, Holodniy M. Oseltamivir for treatment and prophylaxis of influenza infection. Expert Opin Drug Saf (2009) 8:357-71. doi:10.1517/ 14740330902840519

4. Ijichi S, Ijichi N. Too early cure of influenza: recurrence in oseltamivir-treated children. J Paediatr Child Health (2003) 39:480-1. doi:10.1046/j.1440-1754. 2003.00197.x

5. Correia V, De Andrade HR, Santos LA, Lackenby A, Zambon M. Antiviral drug profile of seasonal influenza viruses circulating in Portugal from 2004/2005 to $2008 / 2009$ winter seasons. Antiviral Res (2010) 86:128-36. doi:10.1016/j. antiviral.2010.01.002

6. Chen LF, Dailey NJM, Rao AK, Fleischauer AT, Greenwald I, Deyde VM, et al. Cluster of oseltamivir-resistant 2009 pandemic influenza A (H1N1) virus influenza pandemic where the only seasonal influenza vaccine that was available failed to induce immune responses capable of protecting individuals against the emergent strain (30). Intranasal administration of PEG-Pam ${ }_{2}$ Cys at such times could provide the population with immediate protection and reduce transmission of virus (1). As we show in this study, individuals treated with $\mathrm{Pam}_{2} \mathrm{Cys}$ and subsequently challenged with virus would develop influenza-specific adaptive immune responses providing longterm protection and removing the need for rapid vaccine production. Another feature of PEG-Pam ${ }_{2}$ Cys as an immunostimulatory agent is that it has the potential to be self-administered reducing the impact placed on medical staff during pandemics.

We have shown that the TLR-2 agonist PEG-Pam ${ }_{2}$ Cys provides mice with immediate protection against influenza virus and does not impact the induction of influenza-specific immunity following subsequent exposure to virus, which provides both homotypic and heterosubtypic protection. The data generated in this study encourages the development of immunostimulatory agents and could also alter our perception of the role that these anti-microbial agents play in long-term immunity to respiratory infections. In the absence of an effective vaccine, the use of $\mathrm{Pam}_{2}$ Cys can immediately reduce the impact of infectious agents and provide an individual with long-lasting immunity through natural infection.

\section{Acknowledgments}

This work was supported by project grant APP1063209 and program grant APP1071916 from the National Health and Medical Research Council of Australia. DCJ is supported by a Research Fellowship from the NH\&MRC. We would like to thank Dr. Weiguang Zeng for technical assistance.

\section{Supplementary Material}

The Supplementary Material for this article can be found online at http://journal.frontiersin.org/article/10.3389/fimmu.2015.00290/ abstract

infections on a hospital ward among immunocompromised patients - North Carolina, 2009. J Infect Dis (2011) 203:838-46. doi:10.1093/infdis/jiq124

7. Hurt AC, Hardie K, Wilson NJ, Deng YM, Osbourn M, Gehrig N, et al. Community transmission of oseltamivir-resistant A(H1N1)pdm09 influenza. N Engl J Med (2011) 365:2541-2. doi:10.1056/NEJMc1111078

8. Tuvim MJ, Evans SE, Clement CG, Dickey BF, Gilbert BE. Augmented lung inflammation protects against influenza a pneumonia. PLoS One (2009) 4:e4176. doi:10.1371/journal.pone.0004176

9. Lau Y-F, Tang L-H, Ooi E-E, Subbarao K. Activation of the innate immune system provides broad-spectrum protection against influenza A viruses with pandemic potential in mice. Virology (2010) 406:80-7. doi:10.1016/j.virol.2010. 07.008

10. Norton EB, Clements JD, Voss TG, Cardenas-Freytag L. Prophylactic administration of bacterially derived immunomodulators improves the outcome of influenza virus infection in a murine model. J Virol (2010) 84:2983-95. doi:10. 1128/jvi.01805-09

11. De St Groth SF, Webster RG. Disquisition on originial antigenic sin. J Exp Med (1966) 124:331-45. doi:10.1084/jem.124.3.331

12. Stambas J, Doherty PC, Turner SJ. An in vivo cytotoxicity threshold for influenza A virus-specific effector and memory CD8+ T cells. J Immunol (2007) 178:1285-92. doi:10.4049/jimmunol.178.3.1285 
13. Tan ACL, Deliyannis G, Bharadwaj M, Brown LE, Zeng W, Jackson DC. The design and proof of concept for a CD8(+) T cell-based vaccine inducing cross-subtype protection against influenza A virus. Immunol Cell Biol (2013) 91:96-104. doi:10.1038/icb.2012.54

14. Tannock GA, Paul JA, Barry RD. Relative immunogenicity of the coldadapted influenza virus A/Ann Arbor/6/60 (A/AA/6/60-ca), recombinants of A/AA/6/60-ca, and parental strains with similar surface antigens. Infect Immun (1984) 43:457-62.

15. Flynn KJ, Belz GT, Altman JD, Ahmed R, Woodland DL, Doherty PC. Virusspecific CD8+ T cells in primary and secondary influenza pneumonia. Immunity (1998) 8:683-91. doi:10.1016/S1074-7613(00)80573-7

16. Topham D, Tripp R, Doherty P. CD $8+$ T cells clear influenza virus by perforin or Fas-dependent processes. J Immunol (1997) 159:5197-200.

17. Chen HD, Fraire AE, Joris I, Brehm MA, Welsh RM, Selin LK. Memory CD8+ $\mathrm{T}$ cells in heterologous antiviral immunity and immunopathology in the lung. Nat Immunol (2001) 2:1067-76. doi:10.1038/ni727

18. Topham DJ, Castrucci MR, Wingo FS, Belz GT, Doherty PC. The role of antigen in the localization of naive, acutely activated, and memory CD8+ T cells to the lung during influenza pneumonia. J Immunol (2001) 167:6983-90. doi:10.4049/ jimmunol.167.12.6983

19. Ely KH, Cauley LS, Roberts AD, Brennan JW, Cookenham T, Woodland DL. Nonspecific recruitment of memory CD8+ T cells to the lung airways during respiratory virus infections. J Immunol (2003) 170:1423-9. doi:10.4049/ jimmunol.170.3.1423

20. Kundig TM, Bachmann MF, Ohashi PS, Pircher H, Hengartner H, Zinkernagel RM. On T cell memory: arguments for antigen dependence. Immunol Rev (1996) 150:63-90. doi:10.1111/j.1600-065X.1996.tb00696.x

21. Gray D. Opinion: a role for antigen in the maintenance of immunological memory. Nat Rev Immunol (2002) 2:60. doi:10.1038/nri706

22. Baaten BJG, Clarke B, Strong P, Hou S. Nasal mucosal administration of chitin microparticles boosts innate immunity against influenza A virus in the local pulmonary tissue. Vaccine (2010) 28:4130-7. doi:10.1016/j.vaccine.2010. 04.026

23. Cottalorda A, Verschelde C, Marçais A, Tomkowiak M, Musette P, Uematsu $\mathrm{S}$, et al. TLR2 engagement on CD8 $\mathrm{T}$ cells lowers the thresholdfor optimal antigen-induced T cell activation. Eur J Immunol (2006) 36:1684-93. doi:10. 1002/eji.200636181

24. Mercier BC, Cottalorda A, Coupet C-A, Marvel J, Bonnefoy-Bérard N. TLR2 engagement on CD8 $\mathrm{T}$ cells enables generation of functional memory cells in response to a suboptimal TCR signal. J Immunol (2009) 182:1860-7. doi:10. 4049/jimmunol.0801167

25. Badovinac VP, Porter BB, Harty JT. CD8+ T cell contraction is controlled by early inflammation. Nat Immunol (2004) 5:809-17. doi:10.1038/ni1098

26. Kohlmeier JE, Reiley WW, Perona-Wright G, Freeman ML, Yager EJ, Connor $\mathrm{LM}$, et al. Inflammatory chemokine receptors regulate CD8+ T cell contraction and memory generation following infection. J Exp Med (2011) 208:1621-34. doi:10.1084/jem.20102110

27. Raué H-P, Beadling C, Haun J, Slifka MK. Cytokine-mediated programmed proliferation of virus-specific CD8+ memory $\mathrm{T}$ cells. Immunity (2013) 38:131-9. doi:10.1016/j.immuni.2012.09.019

28. Curtsinger JM, Johnson CM, Mescher MF. CD8 T cell clonal expansion and development of effector function require prolonged exposure to antigen, costimulation, and signal 3 cytokine. J Immunol (2003) 171:5165-71. doi:10.4049/ jimmunol.171.10.5165

29. Richer MJ, Nolz JC, Harty JT. Pathogen-specific inflammatory milieux tune the antigen sensitivity of CD8 $+\mathrm{T}$ cells by enhancing $\mathrm{T}$ cell receptor signaling. Immunity (2013) 38:140-52. doi:10.1016/j.immuni.2012.09.017

30. Hancock K, Veguilla V, Lu X, Zhong W, Butler EN, Sun H, et al. Cross-reactive antibody responses to the 2009 pandemic H1N1 influenza virus. N Engl J Med (2009) 361:1945-52. doi:10.1056/NEJMoa0906453

Conflict of Interest Statement: The authors declare that the research was conducted in the absence of any commercial or financial relationships that could be construed as a potential conflict of interest.

Copyright (c) 2015 Mifsud, Tan, Brown, Chua and Jackson. This is an open-access article distributed under the terms of the Creative Commons Attribution License (CC $B Y)$. The use, distribution or reproduction in other forums is permitted, provided the original author(s) or licensor are credited and that the original publication in this journal is cited, in accordance with accepted academic practice. No use, distribution or reproduction is permitted which does not comply with these terms. 